\title{
Electrically charged dilaton black holes in external magnetic field
}

\author{
Stoytcho S. Yazadjiev * \\ Department of Theoretical Physics, Faculty of Physics, Sofia University, \\ 5 James Bourchier Boulevard, Sofia 1164, Bulgaria \\ and \\ Theoretical Astrophysics, Eberhard-Karls University of Tübingen, Tübingen 72076, Germany
}

\begin{abstract}
In the present paper we construct a new solution to the Einstein-Maxwelldilaton gravity equations describing electrically charged dilaton black holes immersed in a strong external magnetic field and we study its properties. The black holes described by the solution are rotating but with zero total angular momentum and possess an ergoregion confined in a neighborhood of the horizon. Our results also show that the external magnetic field does not affect the black hole thermodynamics.
\end{abstract}

\section{Introduction}

Dilaton black holes have been extensively studied in various aspects during the last two decades. Nevertheless, there still remain open problems which are not solved up to now. The present paper is devoted to one such problem, namely the construction of exact solutions describing electrically charged dilaton black holes in a strong external magnetic field and the study of their properties. In contrast to the exact solutions describing uncharged dilaton black holes in external magnetic field, the construction of electrically charged black holes in external magnetic field is much more difficult. The reason hides behind the fact that such solutions have to be in principle stationary due to the contribution of electromagnetic circulating momentum flux $E \wedge B$ in the energymomentum tensor. It is well known, however, that the construction of rotating black holes in Einstein-Maxwell-dilaton (EMd) gravity is extremely difficult for arbitrary dilaton coupling parameter. In fact, the only known rotating black hole solution in EMd gravity is the black hole with Kaluza-Klein coupling $\alpha=\sqrt{3}[1,22$. The finding of rotating EMd black holes with arbitrary dilaton coupling parameter is another yet unsolved problem. In view of the described difficulties we will consider the EMd gravity with $\alpha=\sqrt{3}$.

*E-mail: yazad@phys.uni-sofia.bg 
The study of black holes in a magnetic field (the so-called magnetized black holes) has a long history starting with the classical papers by Wald [3] and Ernst [4]. Afterwards the magnetized black holes within general relativity and other alternatives theories were studied by many authors in various aspects ranging from pure theoretical studies to astrophysics [5]-23. The thermodynamics of the magnetized black holes is of special interest. The naive expectation was that a thermodynamic description of these configurations would include also the value of the background magnetic field as a further parameter. However, more detailed studies showed that this was not the case for a static electrically uncharged solutions in four dimensions and some rotating black holes in higher dimensions - the external magnetic field only distorts the horizon geometry without affecting the thermodynamics [19], [20], [23]. The thermodynamics of electrically charged or/and rotating magnetized black holes is not fully studied and it is not clear whether the magnetic field affects it or not. The situation with the thermodynamics of electrically charged and/or rotating magnetized black holes seems very complicated in the light of the very recent result of [27. Unexpectedly, it was discovered in 27] that the magnetized electrically charged Reissner-Nordström and Kerr-Newman black holes are not asymptotic to the static Melvin solution [24]. Even more surprising, it was shown in [27] that, in the general case, the ergoregion of the magnetized Reissner-Nordström and Kerr-Newman black holes extends all the way from the horizon to infinity. The natural question is whether a similar behavior occurs for other electrically charged black holes. In the present paper we give also a partial answer to this question for some black holes in EMd gravity.

\section{Construction of the exact solution}

The field equations of the four-dimensional Einstein-Maxwell-dilaton gravity are given by

$$
\begin{aligned}
& R_{\mu \nu}=2 \nabla_{\mu} \varphi \nabla_{\nu} \varphi+2 e^{-2 \alpha \varphi}\left(F_{\mu \sigma} F_{\nu}{ }^{\sigma}-\frac{g_{\mu \nu}}{4} F_{\rho \sigma} F^{\rho \sigma}\right), \\
& \nabla_{\mu}\left(e^{-2 \alpha \varphi} F^{\mu \nu}\right)=0=\nabla_{[\mu} F_{\nu \sigma]}, \\
& \nabla_{\mu} \nabla^{\mu} \varphi=-\frac{\alpha}{2} e^{-2 \alpha \varphi} F_{\rho \sigma} F^{\rho \sigma},
\end{aligned}
$$

where $\nabla_{\mu}$ and $R_{\mu \nu}$ are the Levi-Civita connection and the Ricci tensor with respect to the spacetime metric $g_{\mu \nu} . F_{\mu \nu}$ is the Maxwell tensor and the dilaton field is denoted by $\varphi$, with $\alpha$ being the dilaton coupling parameter governing the coupling strength of the dilaton to the electromagnetic field.

We shall consider spacetimes admitting a spacelike Killing field $\eta$ with closed orbits. The dimensional reduction of the field equations along the Killing field was performed in [25. So we present here only the basic steps skipping the details which can be found in [25].

Since the Maxwell 2-form $F$ is invariant under the flow of the Killing field there exist potentials $\Phi$ and $\Psi$ defined by $d \Phi=i_{\eta} F$ and $d \Psi=-e^{-2 \alpha \varphi} i_{\eta} \star F$ such that 


$$
F=X^{-1} \eta \wedge d \Phi-X^{-1} e^{2 \alpha \varphi} \star(d \Psi \wedge \eta)
$$

where

$$
X=g(\eta, \eta) \text {. }
$$

The twist $\omega$ of the Killing field $\eta$, defined by $\omega=\star(d \eta \wedge \eta)$, satisfies the equation

$$
d \omega=4 d \Psi \wedge d \Phi=d(2 \Psi d \Phi-2 \Phi d \Psi) .
$$

Hence we conclude that there exists a twist potential $\chi$ such that $\omega=d \chi+2 \Psi d \Phi-$ $2 \Phi d \Psi$.

The projection metric $\gamma$ orthogonal to the Killing field $\eta$, is defined by

$$
g=X^{-1}(\gamma+\eta \otimes \eta) .
$$

In local coordinates adapted to the Killing field, i.e. $\eta=\partial / \partial \phi$, we have

$$
d s^{2}=g_{\mu \nu} d x^{\mu} d x^{\nu}=X\left(d \phi+W_{a} d x^{a}\right)^{2}+X^{-1} \gamma_{a b} d x^{a} d x^{b} .
$$

The 1-form $W=W_{a} d x^{a}$ is closely related to the twist $\omega$ which can be expressed in the form

$$
\omega=X i_{\eta} \star d W
$$

The dimensionally reduced EMd equations form an effective 3-dimensional gravity coupled to a nonlinear $\sigma$-model with the following action

$$
\mathcal{A}=\int d^{3} x \sqrt{-\gamma}\left[R(\gamma)-2 \gamma^{a b} G_{A B} \partial_{a} X^{A} \partial_{b} X^{B}\right]
$$

where $R(\gamma)$ is the Ricci scalar curvature with respect to the metric $\gamma_{a b}, X^{A}=$ $(X, \chi, \Phi, \Psi, \varphi)$ and $G_{A B}$ can be viewed as a metric on an abstract Riemannian manifold $\mathcal{N}$ with local coordinates $X^{A}$ and its explicit form is given by

$$
G_{A B} d X^{A} d X^{B}=\frac{d X^{2}+(d \chi+2 \Phi d \Psi-2 \Psi d \Phi)^{2}}{4 X^{2}}+\frac{e^{-2 \alpha \varphi} d \Phi^{2}+e^{2 \alpha \varphi} d \Psi^{2}}{X}+d \varphi^{2} .
$$

What is important for the aim of the present paper is the fact that the Riemannian space $\left(\mathcal{N}, G_{A B}\right)$ is a symmetric space for the critical coupling $\alpha=\sqrt{3}$. In fact $\mathcal{N}$ is an $S L(3, R) / O(3)$ symmetric space and therefore its metric can be written in the form

$$
G_{A B} d X^{A} d X^{B}=\frac{1}{8} \operatorname{Tr}\left(S^{-1} d S S^{-1} d S\right)
$$


where $S$ is a symmetric $S L(3, R)$ matrix explicitly given by [26]

$$
S=e^{-\frac{2}{3} \sqrt{3} \varphi}\left(\begin{array}{ccc}
X^{-1} & -2 X^{-1} \Phi & X^{-1}(2 \Phi \Psi-\chi) \\
-2 X^{-1} \Phi & e^{2 \sqrt{3} \varphi}+4 X^{-1} \Phi^{2} & -2 e^{2 \sqrt{3} \varphi} \Psi-2 X^{-1}(2 \Phi \Psi-\chi) \Phi \\
X^{-1}(2 \Phi \Psi-\chi) & -2 e^{2 \sqrt{3} \varphi} \Psi-2 X^{-1}(2 \Phi \Psi-\chi) \Phi & X+4 \Psi^{2} e^{2 \sqrt{3} \varphi}+X^{-1}(2 \Phi \Psi-\chi)^{2}
\end{array}\right)
$$

The group of symmetries $S L(3, R)$ can be used to generate new solutions from known ones via the scheme

$$
S \rightarrow \Gamma S \Gamma^{T}, \gamma_{a b} \rightarrow \gamma_{a b}
$$

where $\Gamma \in S L(3, R)$. In the present paper we consider seed solutions corresponding to the matrix

$$
S_{0}=e^{-\frac{2}{3} \sqrt{3} \varphi_{0}}\left(\begin{array}{ccc}
X_{0}^{-1} & 0 & 0 \\
0 & e^{2 \sqrt{3} \varphi_{0}} & -2 e^{2 \sqrt{3} \varphi_{0}} \Psi_{0} \\
0 & -2 e^{2 \sqrt{3} \varphi_{0}} \Psi_{0} & X_{0}+4 \Psi_{0}^{2} e^{2 \sqrt{3} \varphi_{0}}
\end{array}\right)
$$

and transformation matrices in the form

$$
\Gamma=\left(\begin{array}{lll}
1 & B & 0 \\
0 & 1 & 0 \\
0 & 0 & 1
\end{array}\right)
$$

with $B$ being an arbitrary real number. In the particular case when the seed solution is a pure Einstein black hole solution, i.e. $\Psi_{0}=0$ and $\varphi_{0}=0$, the above transformation gives the solution describing uncharged black holes in external magnetic field [23]. The physical meaning of the parameter $B$ is the asymptotic strength of the external magnetic field.

The new solutions which can be generated from the seed and the transformation matrices under consideration, are encoded in the matrix $S=\Gamma S_{0} \Gamma^{T}$ and the explicit form of their potentials is as follows

$$
\begin{aligned}
& X=\frac{X_{0}}{\sqrt{1+B^{2} e^{2 \sqrt{3} \varphi_{0} X_{0}}}}, \\
& e^{\frac{4}{\sqrt{3}} \varphi}=\frac{e^{\frac{4}{\sqrt{3}} \varphi_{0}}}{1+B^{2} e^{2 \sqrt{3} \varphi_{0}} X_{0}} \\
& \Phi=-\frac{B}{2} \frac{e^{2 \sqrt{3} \varphi_{0}} X_{0}}{1+B^{2} e^{2 \sqrt{3} \varphi_{0}} X_{0}}, \\
& \Psi=\Psi_{0}, \\
& \chi=-2 \Psi_{0} \Phi .
\end{aligned}
$$


In order to find the solution describing an electrically charged dilaton black hole in external magnetic field we choose the seed solution to be the electrically charged, static and spherically symmetric dilaton black hole solution with $\alpha=\sqrt{3}$ [28],[29], namely

$$
\begin{aligned}
& d s_{0}^{2}=-\frac{1-\frac{r_{+}}{r}}{\sqrt{1-\frac{r_{-}}{r}}} d t^{2}+\frac{\sqrt{1-\frac{r_{-}}{r}}}{1-\frac{r_{+}}{r}} d r^{2}+r^{2}\left(1-\frac{r_{-}}{r}\right)^{3 / 2}\left(d \theta^{2}+\sin ^{2} \theta d \phi^{2}\right), \\
& e^{2 \sqrt{3} \varphi_{0}}=\left(1-\frac{r_{-}}{r}\right)^{3 / 2}, \\
& F_{0}=-\frac{q}{r^{2}} d t \wedge d r .
\end{aligned}
$$

The relation between the mass and the charge of the seed solution with the parameters $r_{+}$and $r_{-}$is given by the formulae

$$
m=\frac{1}{2}\left(r_{+}-\frac{r_{-}}{2}\right), \quad q^{2}=\frac{r_{+} r_{-}}{4} .
$$

Using the definition of the potential $\Psi$ we find that the potential $\Psi_{0}$ corresponding to the seed solution is given by

$$
\Psi_{0}=-q \cos \theta
$$

The only two quantities that should be found in order to obtain the new solution describing an electrically charged dilaton black hole in an external magnetic field, are the rotational 1-form $W$ and the Maxwell 2-form $F$. The rotational 1-form $W$ can be found by reversing eq.(9)

$$
d W=X^{-2} i_{\eta} \star \omega
$$

and taking into account that for the new solution $\omega=-4 \Phi d \Psi_{0}$. After some algebra with differential forms we obtain

$$
W=-\frac{2 q B}{r} d t
$$

The Maxwell 2-form can be found in the same way by using eq.(41) and the result is

$$
F=-d\left[\frac{q}{r\left(1+B^{2} e^{2 \sqrt{3} \varphi_{0}} X_{0}\right)}\right] \wedge d t+d \Phi \wedge d \phi,
$$

or equivalently the gauge potential is given by

$$
A=A_{\mu} d x^{\mu}=-\frac{q}{r\left(1+B^{2} e^{2 \sqrt{3} \varphi_{0}} X_{0}\right)} d t+\Phi d \phi
$$


Now we can present the solution in a fully explicit form:

$$
\begin{aligned}
& d s^{2}=\sqrt{1+B^{2} r^{2}\left(1-\frac{r_{-}}{r}\right)^{3} \sin ^{2} \theta}\left[-\frac{1-\frac{r_{+}}{r}}{\sqrt{1-\frac{r_{-}}{r}}} d t^{2}+\frac{\sqrt{1-\frac{r_{-}}{r}}}{1-\frac{r_{+}}{r}} d r^{2}+r^{2}\left(1-\frac{r_{-}}{r}\right)^{3 / 2} d \theta^{2}\right] \\
& +\frac{r^{2}\left(1-\frac{r_{-}}{r}\right)^{3 / 2} \sin ^{2} \theta}{\sqrt{1+B^{2} r^{2}\left(1-\frac{r_{-}}{r}\right)^{3} \sin ^{2} \theta}}\left(d \phi-\frac{2 q B}{r} d t\right)^{2}, \\
& e^{2 \sqrt{3} \varphi}=\left[\frac{1-\frac{r_{-}}{r}}{1+B^{2} r^{2}\left(1-\frac{r_{-}}{r}\right)^{3} \sin ^{2} \theta}\right]^{3 / 2} \\
& \left.A_{t}=-\frac{q}{r\left[1+B^{2} r^{2}\left(1-\frac{r_{-}}{r}\right)^{3} \sin ^{2} \theta\right.}\right] \\
& A_{\phi}=-\frac{B}{2} \frac{r^{2}\left(1-\frac{r_{-}}{r}\right)^{3} \sin ^{2} \theta}{1+B^{2} r^{2}\left(1-\frac{r_{-}}{r}\right)^{3} \sin ^{2} \theta} .
\end{aligned}
$$

In the particular case when $q=0$ the solution reduces to the solution describing the Schwarzschild-dilaton black hole with $\alpha=\sqrt{3}$ in an external magnetic field.

It is interesting to note that our 4-dimensional EMd solution corresponds to a pure vacuum solution to the 5-dimensional Einstein gravity which can be found by performing a Kaluza-Klein uplifting via the equation

$$
d s_{5}^{2}=e^{\frac{2}{3} \sqrt{3} \varphi} d s_{4}^{2}+e^{-\frac{4}{3} \sqrt{3} \varphi}\left(d x_{5}+2 A_{\mu} d x^{\mu}\right)^{2} .
$$

In completely explicit form we have

$$
\begin{gathered}
d s_{5}^{2}=-\left(1-\frac{r_{+}}{r-r_{-}}\right) d t^{2}+\frac{r-r_{-}}{r-r_{+}} d r^{2}+\left(r-r_{-}\right)^{2} d \theta^{2}+\left(r-r_{-}\right)^{2} \sin ^{2} \theta d \phi^{2} \\
+\left[\frac{r}{r-r_{-}}+B^{2}\left(r-r_{-}\right)^{2} \sin ^{2} \theta\right] d x_{5}^{2}-2 \frac{\sqrt{r_{+} r_{-}}}{r-r_{-}} d t d x_{5}-2 B\left(r-r_{-}\right)^{2} \sin ^{2} \theta d \phi d x_{5} .
\end{gathered}
$$

It is also worth noting that there is one more method for generating our EMd solution. It can be generated via a twisted Kaluza-Klein reduction of the 5-dimensional uplifted seed solution

$$
\begin{aligned}
& d s_{5(\text { seed })}^{2}=-\left(1-\frac{r_{+}}{r-r_{-}}\right) d t^{2}+\frac{r-r_{-}}{r-r_{+}} d r^{2}+\left(r-r_{-}\right)^{2} d \theta^{2}+\left(r-r_{-}\right)^{2} \sin ^{2} \theta d \phi^{2} \\
& +\frac{r}{r-r_{-}} d x_{5}^{2}-2 \frac{\sqrt{r_{+} r_{-}}}{r-r_{-}} d t d x_{5}
\end{aligned}
$$

along the Killing field $V=B \frac{\partial}{\partial \phi}+\frac{\partial}{\partial x_{5}}$. Indeed, introducing the new coordinate $\phi_{*}=$ $\phi-B x_{5}$, which is invariant under $V$, we find that (36) transforms to (35) and the further Kaluza-Klein reduction obviously gives our EMd solution. 


\section{Properties of the solution}

In the present section we investigate some of the basic properties of the solution constructed in the previous section.

First we note that our solution is free from conical singularities and the periodicity of $\phi$ is the usual one $\Delta \phi=2 \pi$. In order to see that the parameter $B$ is indeed the asymptotic magnetic field let us calculate $\vec{B}^{2}$ on the axis of axial symmetry. We have

$$
\left.\vec{B}^{2}\right|_{\text {axis }}=B^{2} e^{4 \sqrt{3} \varphi_{0}}=B^{2}\left(1-\frac{r_{-}}{r}\right)^{3}
$$

which shows that $B$ is the asymptotic magnetic field strength in the limit $r \rightarrow \infty$.

There is a Killing horizon at $r=r_{+}$where the Killing field $K=\frac{\partial}{\partial t}+\Omega_{H} \frac{\partial}{\partial \phi}$ becomes null. Here $\Omega_{H}=\frac{2 q B}{r_{+}}$is the angular velocity of the horizon. The metric induced on the horizon cross section is

$d s_{H}^{2}=\sqrt{1+B^{2} r^{2}\left(1-\frac{r_{-}}{r}\right)^{3} \sin ^{2} \theta} r^{2}\left(1-\frac{r_{-}}{r}\right)^{3 / 2} d \theta^{2}+\frac{r^{2}\left(1-\frac{r_{-}}{r}\right)^{3 / 2} \sin ^{2} \theta}{\sqrt{1+B^{2} r^{2}\left(1-\frac{r_{-}}{r}\right)^{3} \sin ^{2} \theta}} d \phi^{2}$.

By applying the Gauss-Bonnet theorem one can show that surface of the event horizon is topologically a 2 -sphere. The horizon area is

$$
\mathcal{A}_{H}=4 \pi r_{+}^{2}\left(1-\frac{r_{-}}{r_{+}}\right)^{3 / 2}
$$

and evidently it coincides with the horizon area of the seed solution. As in the uncharged case, the external magnetic field deforms the horizon but preserves the horizon area. In fact the geometry of the horizon cross section deviates from that of the round 2 -sphere. A simple inspection of (38) reveals that the polar circumference $(\phi=$ const) is greater than the circumference about the equator $\left(\theta=\frac{\pi}{2}\right)$. Therefore, for the solution under consideration the magnetic field elongates the black hole along the magnetic field and the black hole is prolate in shape.

The physical electric charge is given by

$$
Q=\frac{1}{4 \pi} \int_{H} e^{-2 \sqrt{3} \varphi} \star F=\frac{1}{2} \int_{\theta=0}^{\pi} d \Psi=q
$$

and coincides with that of the seed solution.

The ergoregion for the solution under consideration is determined by the region where $g\left(\frac{\partial}{\partial t}, \frac{\partial}{\partial t}\right)=g_{t t}$ is positive. The explicit form of $g_{t t}$ is 


$$
g_{t t}=\frac{-\left(r-r_{+}\right)+\left(r_{+}+r_{-}-r\right)\left(r-r_{-}\right)^{2} B^{2} \sin ^{2} \theta}{r \sqrt{1-\frac{r_{-}}{r}} \sqrt{1+B^{2} r^{2}\left(1-\frac{r_{-}}{r}\right)^{3} \sin ^{2} \theta}}
$$

It is easy to see that very close to the horizon we have $g_{t t}>0$ for $\sin \theta \neq 0$ and $g_{t t}=0$ for $\sin \theta=0$ and $r=r_{+}$. Also, it is not difficult to see that for $r \geq r_{+}+r_{-}$ it holds that $g_{t t}<0$. Therefore, we conclude that there exists an ergoregion confined in a compact neighborhood of the horizon, in contrast with the magnetized ReissnerNordström solution for which the ergorgeon extents to infinity [27]. The boundary of the ergoregion, i.e. the ergosurface, is defined by $g_{t t}=0$ which reduces to a cubic equation in $r$, namely $r-r_{+}=\left(r_{+}+r_{-}-r\right)\left(r-r_{-}\right)^{2} B^{2} \sin ^{2} \theta$. Solving this cubic equation for $r$ we can find the equation $r(\theta)$ of the ergosurface. The explicit form of $r(\theta)$ is too cumbersome to be presented here. What is important is that the ergosurface is qualitatively the same as the ergosurface of the Kerr solution.

The surface gravity $\kappa$ associated with the Killing field $K$ can be calculated via the well-known formula

$$
\kappa^{2}=-\frac{g(d \lambda, d \lambda)}{4 \lambda}
$$

where $\lambda=g(K, K)$. The direct computation gives the following result

$$
\kappa=\frac{1}{2 r_{+} \sqrt{1-\frac{r_{-}}{r_{+}}}}
$$

which is just the surface gravity of the seed solution. Therefore, the surface gravity is not affected by the external magnetic field for the solution under consideration.

The inspection of the electric field shows that $\vec{E}^{2} \rightarrow 0$ for $r \rightarrow \infty$. The same holds for the norm of the twist of the Killing vectors $\eta=\frac{\partial}{\partial \phi}$ and $\xi=\frac{\partial}{\partial t}$. As a whole, our solution is asymptotic to the dilaton-Melvin solution with $\alpha=\sqrt{3}$. This can be easily seen from its explicit form.

\section{Thermodynamics}

The study of the thermodynamics of the black holes in external magnetic fields is difficult because of the asymptotic structure. Since the spacetime is not asymptotically flat a substraction procedure is needed to obtain finite quantities from integrals divergent at infinity. The natural choice for the substraction background in our case is the dilaton-Melvin background. To calculate the mass we use the quasilocal formalism [23]. Here we give for completeness a very brief description of the quasilocal formalism.

The spacetime metric is decomposed into the form

$$
d s^{2}=-N^{2} d t^{2}+h_{i j}\left(d x^{i}+N^{i} d t\right)\left(d x^{j}+N^{j} d t\right)
$$


with $N$ and $N^{i}$ being the lapse function and the shift vector. The decomposition means that the spacetime is foliated by spacelike surfaces $\Sigma_{t}$ of metric $h_{\mu \nu}=g_{\mu \nu}+u_{\mu} u_{\nu}$, labeled by a time coordinate $t$ with a unit normal vector $u^{\mu}=-N \delta_{0}^{\mu}$. The spacetime boundary consists of the initial surface $\Sigma_{i}\left(t=t_{i}\right)$, the final surface $\Sigma_{f}\left(t=t_{f}\right)$ and a timelike surface $\mathcal{B}$ to which the vector $u^{\mu}$ is tangent. The surface $\mathcal{B}$ is foliated by 2-dimensional surfaces $S_{t}^{r}$, with metric $\sigma_{\mu \nu}=h_{\mu \nu}-n_{\mu} n_{\nu}$, which are the intersections of $\Sigma_{t}$ and $\mathcal{B}$. The unit spacelike outward normal to $S_{t}^{r}, n_{\mu}$, is orthogonal to $u^{\mu}$.

In order to have well-defined variational principle we must consider the extended EMd action with the corresponding boundary terms added:

$$
\begin{array}{r}
S=\frac{1}{16 \pi} \int d^{4} x \sqrt{-g}\left(R-2 g^{\mu \nu} \partial_{\mu} \varphi \partial_{\nu} \varphi-e^{-2 \alpha \varphi} F^{\mu \nu} F_{\mu \nu}\right) \\
+\frac{1}{8 \pi} \int_{\Sigma_{i}}^{\Sigma_{f}} K \sqrt{h} d^{3} x-\frac{1}{8 \pi} \int_{\mathcal{B}} \Theta \sqrt{\sigma} d^{2} x .
\end{array}
$$

Here $K$ is the trace of the extrinsic curvature $K^{\mu \nu}$ of $\Sigma_{t_{i, f}}$ and $\Theta$ is the trace of the extrinsic curvature $\Theta^{\mu \nu}$ of $\mathcal{B}$, given by

$$
\begin{aligned}
K_{\mu \nu} & =-\frac{1}{2 N}\left(\frac{\partial h_{\mu \nu}}{\partial t}-2 D_{(\mu} N_{\nu)}\right) \\
\Theta_{\mu \nu} & =-h_{\mu}^{\alpha} \nabla_{\alpha} n_{\nu}
\end{aligned}
$$

where $\nabla_{\mu}$ and $D_{\nu}$ are the covariant derivatives with respect to the metric $g_{\mu \nu}$ and $h_{\mu \nu}$, respectively.

The quasilocal energy $M$ and the angular momentum $J_{i}$ are given by

$$
\begin{array}{r}
M=\frac{1}{8 \pi} \int_{S_{t}^{r}} \sqrt{\sigma}\left[N\left(k-k_{0}\right)+\frac{n_{\mu} p^{\mu \nu} N_{\nu}}{\sqrt{h}}\right] d^{D-2} x \\
+\frac{1}{4 \pi} \int_{S_{t}^{r}} A_{0}\left(\hat{\Pi}^{j}-\hat{\Pi}_{0}^{j}\right) n_{j} d^{D-2} x \\
J_{i}=-\frac{1}{8 \pi} \int_{S_{t}^{r}} \frac{n_{\mu} p_{i}^{\mu}}{\sqrt{h}} \sqrt{\sigma} d^{D-2} x-\frac{1}{4 \pi} \int_{S_{t}^{r}} A_{i} \hat{\Pi}^{j} n_{j} d^{D-2} x
\end{array}
$$

Here $k=-\sigma^{\mu \nu} D_{\nu} n_{\mu}$ is the trace of the extrinsic curvature of $S_{t}^{r}$ embedded in $\Sigma_{t}$. The momentum variable $p^{i j}$ conjugated to $h_{i j}$ is given by

$$
p^{i j}=\sqrt{h}\left(h^{i j} K-K^{i j}\right)
$$

The quantity $\hat{\Pi}^{j}$ is defined by

$$
\hat{\Pi}^{j}=-\frac{\sqrt{\sigma}}{\sqrt{h}} \sqrt{-g} e^{-2 \alpha \varphi} F^{0 j} .
$$

The quantities with the subscript " 0 " are those associated with the background. 
After long calculations, for the quasilocal energy of the black hole we find

$$
M=m=\frac{1}{2}\left(r_{+}-\frac{1}{2} r_{-}\right)
$$

which is evidently independent from the external magnetic field and coincides with the mass of the seed solution. In the same way, for the angular momentum we obtain

$$
J=0 .
$$

Even more, the angular momentum $J_{S_{t}^{r}}$ associated with every surface $S_{t}^{r}$ is zero. So we have a rotating black hole (i.e. $\Omega_{H} \neq 0$ ) while the total angular momentum is zero. This, at first sight strange result, means that the gravitational contribution to the angular momentum is exactly compensated by the opposite in sign angular momentum of the electromagnetic field.

Furthermore, the following Smarr-like relation is satisfied

$$
M=\frac{1}{4 \pi} \kappa \mathcal{A}_{H}+\Xi_{H} Q,
$$

where the potential $\Xi_{H}$ is in fact the corotating electric potential evaluated on the horizon and given by

$$
\Xi_{H}=-\left.K^{\mu} A_{\mu}\right|_{H}=-\left.\left(A_{t}+\Omega_{H} A_{\phi}\right)\right|_{H}=\frac{Q}{r_{+}} .
$$

On the basis of the results obtained so far we can conclude that the external magnetic field does not affect the thermodynamics of the charged dilaton black holes.

\section{Discussion}

In the present paper we constructed a new solution to the EMd gravity equations describing charged dilaton black holes in external magnetic field for dilaton coupling parameter $\alpha=\sqrt{3}$. The basis properties of the solution and its thermodynamics were studied. The black holes described by the solution are rotating but with zero total angular momentum and possess an ergoregion confined in a neighborhood of the horizon. Our results also show that the external magnetic field does not affect the black hole thermodynamics.

The natural generalization of this work is to consider electrically charged and rotating dilaton black holes immersed in an external magnetic field and to study their thermodynamics. Especially, it is interesting whether the thermodynamics of the rotating solutions depends nontrivially on the external magnetic field. Other interesting questions are the asymptotic structure at infinity and the compactness or non-compactness of the ergoregion. These problems are currently under investigation and the results will be presented elsewhere.

\section{Acknowledgments}


The author is grateful to the Research Group Linkage Programme of the Alexander von Humboldt Foundation for the support of this research and the Institut für Theoretische Astrophysik Tübingen for its kind hospitality. He also acknowledges partial support from the Bulgarian National Science Fund under Grant DMU-03/6.

\section{References}

[1] V. Frolov, A Zelnikov and U. Bleyer, Ann. Phys. 44, 371 (1987).

[2] D. Rasheed, Nucl. Phys. B454, 379 (1995).

[3] R. Wald, Phys. Rev. D10, 1680 (1974).

[4] F. Ernst, J. Math. Phys. 17, 54 (1976).

[5] F. Ernst and W. Wild, J. Math. Phys. 17, 182 (1976).

[6] W. Wild and R. Kerns, Phys. Rev. D21, 332 (1980).

[7] W. Hiscock, J. Math. Phys. 22, 1828 (1981).

[8] S. Bose, and E.Esteban, J. Math. Phys. 22, 3006 (1981).

[9] J. Bicak and V. Janis, Mon. Not. R. Astron. Soc. 212, 899 (1985).

[10] A. Garcia Diazt, J. Math. Phys. 26, 155 (1985).

[11] G. Gibbons and D. Wiltshire, Nucl. Phys. B287, 717 (1987).

[12] V. Karas, Bull. Astron. Inst. Czechosl. 39, 30 (1988).

[13] A. Aliev and D. Gal'tsov, Astrophys. Space Sci. 155, 181 (1989).

[14] V. Karas and D. Vokrouhlicky, J. Math. Phys. 32, 714 (1991).

[15] V. Karas and D. Vokrouhlicky, Gen. Rel. Grav. 24, 729 (1992).

[16] F. Dowker, J. Gauntlett, D. Kastor and J. Traschen, Phys. Rev. D50, 2662 (1994).

[17] A. Chamblin, R. Emparan and G. Gibbons, Phys. Rev. D58, 084009 (1998).

[18] V. Karas and Z. Budinova, Phys. Scr. 61, 253 (2000).

[19] E. Radu, Mod. Phys. Lett. A17, 2277 (2002).

[20] M. Ortaggio, Phys. Rev. D69, 064034 (2004).

[21] A. Aliev and V. Frolov, Phys. Rev. D69, 084022 (2004).

[22] M. Ortaggio, JHEP 0505, 048 (2005).

[23] S. Yazadjiev, Phys. Rev. D73, 064008 (2006). 
[24] M. Melvin, Phys. Rev. 139, B225 (1965).

[25] S. Yazadjiev, Phys. Rev. D82, 124050 (2010).

[26] S. Yazadjiev, Phys. Rev. D87, 024016 (2013).

[27] G. Gibbons, A. Mujtaba and C.Pope, arXiv:1301.3927[gr-qc]

[28] G. Gibbons and K. Maeda, Nucl. Phys. B298, 741 (1988).

[29] D. Garfinkle, G. Horowitz and A. Strominger, Phys. Rev. D43, 3140 (1991); D45, 3888, $1992(\mathrm{E})$. 